Review

\title{
Dysphagia in Inclusion Body Myositis, A Disease of the Elderly
}

Namita A. Goyal ${ }^{1,}{ }^{*}$, Tahseen Mozaffar ${ }^{1,2,3}$

1. Department of Neurology, University of California, Irvine, CA, USA; E-Mails: namitag@hs.uci.edu; mozaffar@hs.uci.edu

2. Department of Orthopaedic Surgery, University of California, Irvine, CA, USA

3. Department of Pathology and Laboratory Medicine, University of California, Irvine, CA, USA

* Correspondence: Namita Goyal; E-Mail: namitag@hs.uci.edu

Academic Editor: David G Smithard

Collection: Dysphagia in the Elderly

OBM Geriatrics

2021, volume 5 , issue 1

doi:10.21926/obm.geriatr.2101154
Received: November 11, 2020

Accepted: January 10, 2021

Published: January 21, 2021

\begin{abstract}
Inclusion body myositis is one of the most common acquired myopathies seen in the aging population. The muscle weakness is of insidious onset, frequently starting in the legs, but over time gradually progresses to eventually involve all skeletal muscles of the limbs, face, swallowing and respiratory muscles, leading to significant morbidity and mortality. Complications resulting from dysphagia are the leading cause of poor quality of life and mortality, while highly prevalent, are still underappreciated and underrecognized. This review highlights the importance of understanding the urgent need for recognizing and improving the evaluation, outcome measures and therapeutic options of dysphagia in inclusion body myositis patients.
\end{abstract}

\section{Keywords}

Inclusion body myositis; dysphagia; aspiration; swallow evaluations; outcome measures

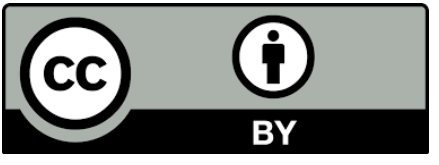

(C) 2021 by the author. This is an open access article distributed under the conditions of the Creative Commons by Attribution License, which permits unrestricted use, distribution, and reproduction in any medium or format, provided the original work is correctly cited. 


\section{Introduction}

Inclusion body myositis (IBM) is one of the most common acquired muscle disorders of the aging population, affecting individuals over the age of 45 years [1, 2]. The disease, which is of insidious onset and gradual progression, causes asymmetric skeletal muscle weakness and atrophy, preferentially affecting the proximal lower extremities (quadriceps) and grip muscles (finger flexors), but over time diffusely affecting all skeletal muscles of the body including the legs, arms, face and even swallowing and diaphragm muscles, leading to severe disability and high morbidity. Patients are often faced with loss of function, impairment of activities of daily living, poor quality of life, as many require a wheelchair for mobility after 10 years from onset, and endure complications resulting from dysphagia [3-5].

The pathogenesis of IBM has yet to be precisely delineated, postulated to have both degenerative and autoimmune features on muscle histopathology, yet refractory to standard immunosuppressive treatments and the disease remains without a cure [6]. As a result, much of the treatment efforts have been directed towards supportive measures with evaluations focused on fall precautions, assistive devices (braces, canes, walkers, wheelchairs), role of exercise, use of non-invasive ventilation to support respiratory insufficiency, and importantly detection and management of dysphagia, as aspiration pneumonia and malnutrition are frequent leading causes of mortality in IBM [7].

\section{Epidemiology of IBM}

The reported incidence of IBM varies from 0.9/million/year to 3.2/million/year depending on the geographic region studied and diagnostic criteria employed [8]. The prevalence estimates have been highly variable ranging from 24.8 to 45.6 per million based on a meta-analysis of published studies [9]. The rarity of the disease and diagnostic challenges has resulted in an average delay from symptom onset to diagnosis of 5.2 years [10], suggesting even the reported prevalence may be an underestimation and likely to increase with improvements in diagnostic criteria, disease awareness, as well as overall due to the aging population.

\section{Mechanism of Dysphagia in IBM}

Dysphagia in a chronic, progressive muscle disease is primarily due to a combination of weakness of several orofacial and pharyngeal muscles. Lip and tongue weakness leads to improper seal and bolus preparation, palatal weakness may result in nasal regurgitation, suprahyoid muscle weakness can cause impaired upper esophageal sphincter opening increasing the risk of aspiration, laryngeal weakness can affect laryngeal closure and coughing, additionally, diaphragm weakness compromises effective coughing and airway clearance [11].

The theories behind the mechanism of dysphagia in IBM are not uniform, but are summarized by 1 ) impaired pharyngeal wall motion due to direct involvement of the pharyngeal musculature 2) impaired laryngeal elevation due to involvement of suprahyoid musculature limiting the opening of the upper esophageal sphincter 3) cricopharyngeal achalasia resulting from inadequate hypopharyngeal 
pressures to trigger reflex relaxation of the muscle and 4) cricopharyngeal hypertrophy and constriction from direct involvement of muscle, causing end-stage fibrosis [12]. Previous histopathological studies evaluating dysphagia in IBM have found that muscle biopsies of the omohyoid and cricopharyngeus muscles show similar findings to that of an IBM limb muscle (quadriceps) with endomysial inflammation, necrotic and regenerating fibers, as well as rimmed vacuoles [13]. These pathologic features would not be anticipated in the physiological effects of aging on the swallowing mechanism.

While all these muscles can weaken in IBM patients, the contribution of impaired cricopharyngeal function and impaired upper esophageal sphincter opening has more recently been implicated as causes of dysphagia in IBM; impaired sphincter function in IBM, a late-onset muscle disease with inflammation and degeneration of the muscles, has also been attributed to the aging process [14]. The reduced upper esophageal opening can lead to pyriform fossa stasis; this sensation of stasis often manifests in an IBM patient as a reported need to swallow repeatedly, food stuck in the throat, the need to use liquids to wash down solids, regurgitation or choking $[15,16]$.

\section{Incidence of Dysphagia and Aspiration in IBM}

Dysphagia is an underrecognized symptom of IBM, present in $10 \%$ to up to $50 \%$ of patients at onset of disease [5-7], and with highly variable incidence over the course of the disease, ranging from $40-80 \%$ $[14,17,18]$. This reported incidence variability is likely secondary to the under-recognition given the insidious onset of symptoms as well as the mechanism used for evaluation (self-report of symptoms, questionnaire, or clinical evaluation). In approximately 6-9\% of IBM patients, dysphagia may be the presenting symptom at onset of disease $[4,19]$, and in some, has even been reported as the sole symptom without limb muscle weakness for up to 5 years [20] adding to the diagnostic delay of IBM, as dysphagia has been under-appreciated as a presenting/prominent feature of IBM. As a result, the prevalence of dysphagia in IBM has been difficult to ascertain and is likely an underestimate.

A study by Cox and colleagues noted symptoms of dysphagia when evaluated via a standard questionnaire were present in 37 of 57 (65\%) IBM patients, yet only $46 \%$ of these patients had previously spontaneously self-reported symptoms of dysphagia, raising concern for under-reporting [14]. Additionally, videofluoroscopy (VFS) detected swallowing abnormalities in $79 \%$ of these patients, highlighting the discrepancy and questioning the diagnostic accuracy between self-reporting and VFS evaluation of dysphagia in IBM [14]. Similar findings were described by Murata et al. who studied dysphagia in two cohorts of IBM patients: those reporting no dysphagia and those reporting symptoms of dysphagia; VFS abnormalities were detected in all IBM patients of both cohorts indicating that the dysphagic process occurs subclinically in those who may not notice swallowing impairments [21].

IBM patients are faced with complications of aspiration pneumonia leading to respiratory failure and death [15, 22]. The evidence of aspiration detected by VFS studies in IBM have ranged from $35 \%$ to as high as $58 \%[14,15,23]$. A study evaluating dysphagia and aspiration in 12 IBM patients reported 7 patients (58\%) experienced aspiration and $3(25 \%)$ had frank aspiration pneumonias [23]. A recent study of 37 IBM patients noted that the presence of a cricopharyngeal bar on VFS contributes to obstruction-related dysphagia and is a risk factor that predicts aspiration pneumonia $(p=0.02)$ [24]. 
Needham and colleagues have described the notable differences in reporting of aspiration among several IBM studies and suspect the figures are more likely in the 50-60\% range [25].

\section{Diagnostic Challenges}

The insidious onset and under-recognition of dysphagia in IBM, often attributed and dismissed as signs of aging, has led to the underreporting and diagnostic challenges. A variety of diagnostic assessments have been studied to evaluate dysphagia in IBM including: direct questions ascertained from the clinical history, screening questionnaires, VFS, flexible endoscopic evaluation of swallowing (FEES), in addition to manometry and MRI (Figure 1). Screening questions obtained from the clinical history are frequently initially used as determining if further investigation (such as VFS) is needed to evaluate for dysphagia. A study by Cox et al. of 57 IBM patients aimed to determine if specific questions from a previously published questionnaire on dysphagia could reliably predict swallowing abnormalities [14]. While the sensitivity and specificity of questions regarding impaired propulsion were suboptimal, two specific questions reliably predicted the presence of impaired propulsion on VFS with high positive predictive values ( 0.92) including: 'does food get stuck in your throat' and 'do you have to swallow repeatedly in order to get rid of food' [14]. Formal standardized patient-based questionnaires can be used as a screening tool in clinic to evaluate dysphagia initially and in follow-up to monitor treatment response. Several standardized swallowing questionnaires are available; of these, the Sydney Swallow Questionnaire and the Swallowing-Related Quality of Life (SWAL-QOL) have demonstrated high validity and reliability for the evaluation of neuromyogenic dysphagia $[26,27]$. Thus, we recommend that patients should perform one of these questionnaires prior to other evaluations in the presence of a caregiver (if present) to improve performance.

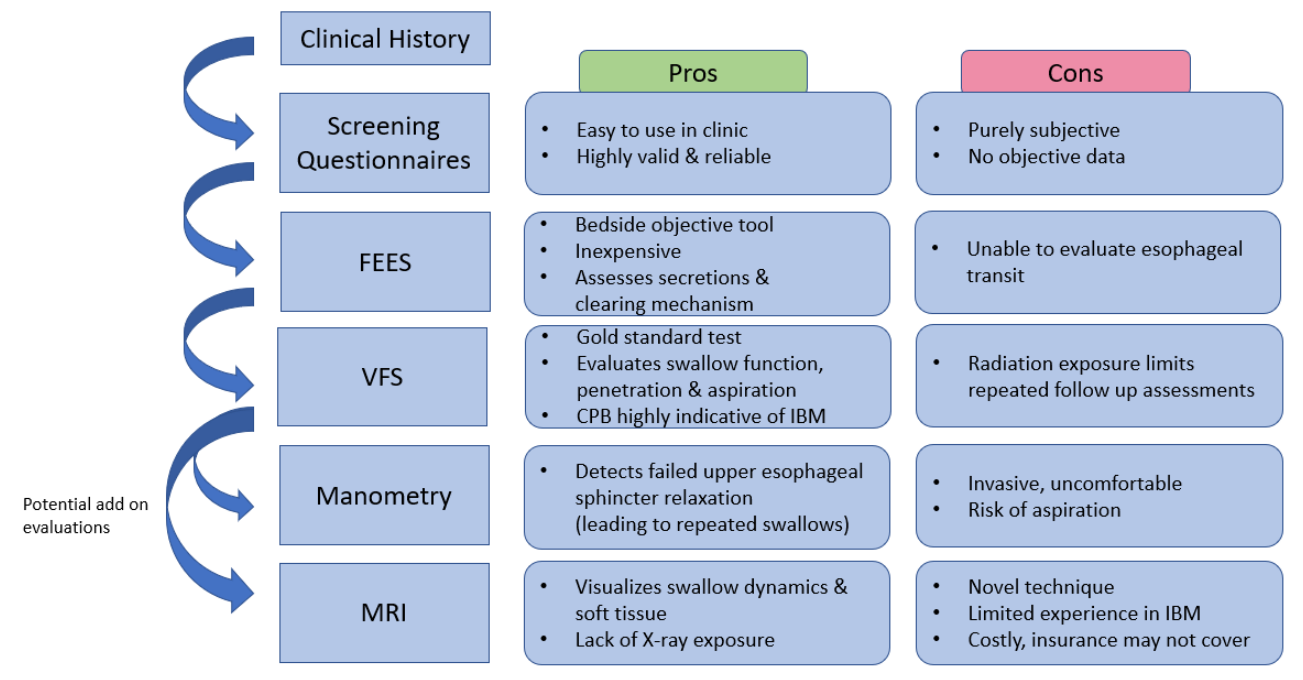

Figure 1 Algorithm of dysphagia evaluation for IBM patients, starting with directed questions in clinical history, leading to screening questionnaires, flexible endoscopic evaluation of swallowing (FEES) at the bedside, to videofluoroscopy (VFS)- a gold standard test. Potential add on evaluations include Manometry and MRI. Pros and cons are listed for each evaluation. $\mathrm{CPB}=$ cricopharyngeal bar. 
Instrumental analysis is considered the gold standard to confirm the diagnosis of dysphagia in IBM and should be performed in any patient whose clinical history suggests swallowing abnormalities; VFS adequately assess swallowing function, penetration and aspiration risk [25]. The most frequent VFS findings when 38 patients with dysphagia were studied included pharyngeal pooling (85\%), impaired tongue base retraction (76\%), impaired laryngeal elevation (50\%), and abnormal cricopharyngeal function (47\%) [7]. The findings of similar abnormalities seen on VFS in IBM reported by other studies $[14,28]$ and the high incidence of detecting dysphagia by VFS (up to $72-79 \%)[14,28]$ suggest that VFS is a reproducible and reliable diagnostic study. A study by Taira et al. demonstrated that against muscular disorders, the finding of the cricopharyngeal bar (CPB) on VFS in IBM narrows the upper esophagus, like a bottleneck, with a sensitivity of $33 \%$ (17/52) yet a high specificity of $96 \%$ (264/274), suggesting the presence of CPB on VFS may be highly indicative of IBM and showing a higher frequency of obstruction-related dysphagia [29]. A limitation of VFS however, is the significant radiation exposure [18].

Flexible (fiberoptic) endoscopic evaluation of swallowing (FEES) is a well-tolerated bedside procedure in which an endoscope is passed from the nasal cavity to the hypopharynx to visualize the larynx and pharynx and to study the physiology of swallowing [30]. It is one of the most commonly used methods for objective assessment of swallowing worldwide [31] and has several advantages due to its practicality including the ability to perform at the bedside, feasibility of follow-up examinations with short-notice, as well as assessment of oropharyngeal secretion management and efficacy of clearing mechanisms [32]. Three components to the FEES examination are: the physical examination of the structural movements, delivery of different foods and liquids, and interventions with dietary or behavioral alterations to address the dysphagia [33]. The FEES Registry study, a large multicenter trial of 2401 patients with dysphagia with a wide-range of primary diagnoses including 29 myopathy patients, confirmed that FEES even when performed by less experienced clinicians was safe, well-tolerated, and in $50 \%$ of the investigations led to changes in feeding strategies, significantly impacting the patients' clinical course and potentially improving dysphagia care [32]. FEES, however, has not been able to replace VFS, as the two procedures provide different clinical information; VFS can reveal a problem with the upper esophageal sphincter opening, esophageal transit, or gastroesophageal reflux disease, whereas, FEES can evaluate laryngeal function and glottic competence to assess change in voice and aspiration during swallow [33]. FEES has not been extensively studied in dysphagia specifically with respect to IBM patients; however a small study of 20 IBM patients found that penetration was seen in $30 \%$ by FEES or VFS and in only $10 \%$ by real-time MRI (RT-MRI) and aspiration was seen in 1 patient by FEES and RT-MRI and in 3 patients by VFS [18]. FEES has gained diagnostic value due to its simplicity, reduced invasiveness, and low cost, but has it's disadvantage due to the known "white out" during the pharyngeal phase, which is typically of great interest in dysphagia [34].

Another diagnostic tool for evaluation of dysphagia has included esophageal manometry which can determine the pharyngeal strength and contraction duration, the completeness of the upper esophageal sphincter relaxation, as well as the coordination between the pharynx and upper esophageal sphincter during swallowing [35]. In a dysphagia study of IBM patients, 12 patients who underwent pharyngoesophageal manometry common findings included: low amplitude pharyngeal constrictor contraction (75\%), normal resting tone and relaxation of the upper esophageal sphincter 
(82\%), and diminished inferior esophageal sphincter pressure (42\%) [15]. Another study of 18 patients with inflammatory myopathies (including 8 with IBM), noted that esophageal manometry is the instrument of choice in demonstrating if the upper esophageal sphincter relaxes prior to swallow triggering, as failed upper esophageal sphincter relaxation may account for dysphagia symptoms such as need for repeated swallows to clear a bolus, choking, or regurgitation; the study recommended that manometry, in combination with VFS, should be performed prior to recommending myotomy [28].

A novel method of real-time MRI (RT-MRI) has been shown in a prior study of healthy individuals as a suitable test for depicting and characterizing the swallowing dynamics [36]. In a study of 20 IBM patients, RT-MRI was equally capable of revealing dysphagia when compared to VFS and FEES and correlated well with the Swallowing-Related Quality of Life Questionnaire (SWAL-QoL) [18]. Additionally, the study suggested that RT-MRI may become a routine diagnostic tool for detailed evaluation dysphagia, as its advantages include precise visualization of soft tissue, more reliable timing analysis and lack of $\mathrm{X}$-ray exposure [18]. Ultrasound evaluation of dysphagia in neuromuscular patients is theoretically a feasible bedside technique that has been reported to detect structural changes in oral and masticatory muscles and can provide dynamic video changes of tongue motion during swallowing attempts [37]; however, there has been limited published literature specifically related to its use in the evaluation of dysphagia in IBM and is currently not used in standard practice.

\section{Treatment Challenges}

There are a number of treatment strategies that have been explored with respect to dysphagia in IBM patients, primarily categorized into medical treatment, rehabilitation measures, and invasive interventional approaches. While several immunosuppressive agents have been tried in IBM, currently no pharmacologic therapy has been proven to be efficacious in slowing or reversing IBM [1]. A number of studies have reported improvement in dysphagia in IBM patients from intravenous immunoglobulin (IVIg) [38-40] as well as in smaller studies using subcutaneous immunoglobulin [41, 42]; however, none of these studies have proved sustained long-term benefit and longer trials are needed to further conclusively determine the role of immunoglobulin therapy with respect to dysphagia.

Swallowing rehabilitation measures, or conservative approaches, that have been evaluated include dietary modifications (as in dietary recommendations of food choices and instruction in feeding techniques), compensatory maneuvers, and swallowing exercises [7]. While positional techniques, such as the chin-tuck can by easy to implement and effective, they are not always sufficient to facilitate a functional swallow, and the use of a swallowing maneuver, such as the Mendelsohn maneuver, a volitional prolongation of laryngeal elevation during midswallow, has been explored [43]. Its use has been shown to increase the duration of upper esophageal sphincter opening and duration of maximal hyoid and laryngeal elevation [44]. Oh et al. evaluated the Mendelsohn maneuver in three IBM patients and reported beneficial effects with all patients maintaining a stable weight and continuing (1-to 5year) ability to eat without aspiration; however, swallowing exercises in 2 IBM patients revealed worsening of symptoms [7]. A recent pilot exercise study by Mohannak et al. explored the efficacy of the Expiratory Muscle Strength Trainer device to see if it would improve hyolaryngeal movement by strengthening suprahyoid muscles, in turn facilitating the opening of the upper esophageal sphincter; 
unfortunately, no overall improvement was seen in swallowing function or quality of life in IBM patients with dysphagia, but further studies will be needed to evaluate the potential benefit noted in those with shorter duration of disease [45].

Botulinum toxin injections to the cricopharyngeal muscle have been investigated as an interventional option in treating dysphagia since 1994 when Schneider et al. treated 7 patients with dysphagia ranging from hypertonicity of the upper esophageal sphincter, complete inability to swallow, and aspiration problems, and found that all but 2 patients experienced complete relief or marked improvement [46]. Since then, a few studies involving a small number of IBM patients (ranging from 212 patients) have reported the beneficial effects of botulinum toxin injections on dysphagia [23, 47, 48]; however, there have been other studies suggesting the lack of improvement or potential complications of transient worsening of dysphagia following the injections $[7,49]$. While the role of botulinum toxin injections for treatment of dysphagia is still unclear, it can be considered for selected patients only in centres with sufficient expertise and larger studies may be needed to further evaluate the safety and efficacy.

Dilation of the pharyngoesophageal muscles and the upper esophageal sphincter dilatation has been tried in IBM patients. Oh and colleagues reported their experience with 26 patients ( 20 of them were women) over a 4 year period and reported that dysphagia in these IBM patients was progressive [15]. Of the 18 patients who underwent intervention, six underwent pharyngoesophageal dilatation and benefit was reported in $33 \%$. Murata et al. reported subjective and objective improvement in dysphagia after balloon catheter dilatation of the upper esophageal sphincter, including barium swallow study evidence of passage of increasing amount of barium paste after dilatation [18]. However, in their study, patients continued to also receive IVIG and it is not clear how much of the benefit was related to the IVIG use.

Surgical division (myotomy) of the cricopharyngeus muscle has been tried in IBM and has been reported with variable success. It has been reported to benefit a large number of patients with IBM and cricopharyngeal dysphagia. Up to sixty percent of the patients report benefit after a myotomy. The myotomy reduces the work of swallowing and reduces the pressure that is required to propel a bolus out of the pharynx. It is, however, irreversible and not all patients with IBM are suited for it. Selection for myotomy needs to be done based on careful study of the mechanisms of swallow dysfunction. Patients with abnormal hyolaryngeal excursion or a hyporeflexic sphincter will not benefit from a cricopharyngeal myotomy [23]. Similarly, patients with hiatal hernia have been shown not to benefit from the myotomy and may result in worsening [50].

Percutaneous endoscopic gastrostomy (PEG) is often used as an intervention of last resort. It is an invasive procedure and does not directly improve swallowing, neither does it influence disease outcome due to the severity of the disease itself. In patients with IBM where swallowing dysfunction is severe enough to cause malnutrition or unintended weight loss that does not respond to other interventions, PEG remains a valid option. However, it is a surgical procedure where the feeding tube has to be inserted through the abdominal wall, commonly requiring sedation and local anesthesia, but at times even deep anesthesia, and there are risks of complications such as pneumonia, bleeding and inadvertent perforation of the stomach or other organs, with resulting peritonitis. Additionally, bloating and osmotic diarrhea from the enteral feeds is quite common and PEG placement does not always 
correct the issues with oral intake. It also does not prevent aspiration and in the Oh et al. study the six patients with IBM who received PEG progressed further and unfortunately died of aspiration pneumonia [15]. However, whilst a PEG tube may not prevent aspiration, it can improve the quality of life for some patients providing adequate nutrition and hydration when the stress of swallowing is high and the burden is bypassed. The role of PEG in IBM at this point is still unclear.

\section{Seropositivity for the NT5c1A Antibody in IBM may Predict Dysphagia}

In 2013, a serum autoantibody binding to the protein NT5c1A, abundant in skeletal muscle with a possible role in DNA repair, was reported initially in IBM patients to have a sensitivity of $60-70 \%$ and a specificity of $83-92 \%[51,52]$, aiding in the diagnostic evaluation of IBM. Our group was interested in evaluating for phenotypic differences between those seropositive versus seronegative for the antiNT5c1A antibody and performed a cross-sectional study of 25 IBM patients, reporting a sensitivity of 72\% for those seropositive [53]. Interestingly, we found that those IBM patients seropositive for the anti-NT5c1A antibody had significantly more motor deficits, bulbar and respiratory involvement, notably with significantly higher odds of having dysphagia $(O R=10.67, p<0.03)$ and facial weakness $(O R=6.0)$ [53]. Subsequently, a large retrospective European study of 311 IBM patients described similar findings in seropositive patients, specifically characterizing a higher likelihood of facial weakness, dysphagia, respiratory events and a significantly higher mortality risk (with a median survival of 17.6 years compared to 24.2 years, seropositive to seronegative, respectively) [54]. These studies have suggested that seropositivity for the NT5C1A antibody in IBM patients may predict a more severe phenotype and incidence of dysphagia. Thus, we recommend those that are seropositive should be routinely screened every 6-12 months for dysphagia.

\section{Outcome Measures for Dysphagia in Clinical Trials of IBM}

Despite the fact that the incidence of dysphagia and dysphagia-associated morbidity and mortality is very high in IBM, the outcome measures in clinical trials that have been developed for IBM are heavily weighted on limb dysfunction and do not provide an exhaustive evaluation of the bulbar dysfunction. Facial weakness is equally common and progressively worsens as disease progresses. However, the current outcome measures do not effectively capture facial weakness or dysphagia. The most commonly used outcome measure is the IBM Functional Rating Scale (IBMFRS), a conglomerate of IBM related symptoms. There is only one question on bulbar function (with a maximum score of 4 out of the total 40 ) that is attributable to swallow function. The IBMFRS recently underwent a Rasch analysis and the discrimination has been improved with division of the scale into upper extremity and lower extremity questions (IBMFRS-UE and IBMFRS-LE) and a reduced 9 question IBMFRS from which the bulbar question was deleted. Thus, the assessment of IBMFRS for limb function has improved with better discrimination, however, has now completely eliminated bulbar function. Novartis developed their own patient-reported outcome measure, sporadic Inclusion Body Myositis Functioning Assessment (SIFA). This was developed in consultation with FDA and met the FDA standards for Patient Reported Outcomes (PROs). This instrument only contains two items (out of eleven) on bulbar dysfunction: one for dysphagia to liquids and one for dysphagia to solids. Additional PROs used for 
dysphagia in IBM have included the SWAL-QOL and EAT-10 questionnaires; however, they are limited by the subjectivity that is inherit in PROs and more objective measures are needed to capture potential changes that may occur in therapeutic trials.

Our group has borrowed the modified oculobulbarfacial respiratory score (mOBFRS) that was developed for Myasthenia Gravis and have adopted it for amyotrophic lateral sclerosis (ALS) as well as inclusion body myositis. This scale has subjective as well as objective elements and evaluates facial and bulbar function, as well as captures a measurement of respiratory function. We performed a study using the MOBFRS scale in ALS patients and have shown the scale has very high sensitivity and specificity and correlates very well with the ALS Functional Rating Scale-Revised (ALSFRS-R) and the bulbar scale that was developed for clinical trials in Kennedy Disease [55]. The mOBFRS data collection and analysis in IBM is still ongoing but are showing similar positive trends in correlation [56].

\section{Conclusion}

Bulbar dysfunction remains a major issue in patients with inclusion body myositis and contributes majorly to the morbidity and mortality of this disease. The dysphagia resulting from this bulbar dysfunction results in weight loss and contributes to the muscle loss. There is also prominent facial weakness that occurs often in parallel to the dysphagia. There is currently a huge unmet need in diagnosis and management of this bulbar dysfunction in IBM. The outcome measures used in research studies of IBM do not adequately capture bulbar dysfunction and there is a need to include more sensitive measures of facial and bulbar dysfunction in IBM research studies, be it interventional or noninterventional. Similarly, there is a need for serial studies of therapeutic intervention for chewing and swallowing dysfunction in IBM and systematic comparative studies of the various interventions and its efficacy in IBM. A transdisciplinary approach involving collaboration between neurologists and dysphagia specialists is needed to properly assess IBM patients for dysphagia. Recognizing the necessity for an improvement in these dysphagia evaluations, outcome measures, and interventions may lead to greater success for drug development and overall quality of life for IBM patients.

\section{Abbreviations}

$\begin{array}{ll}\text { ALS } & \text { Amyotrophic lateral sclerosis } \\ \text { ALSFRS-R } & \text { ALS functional rating scale-revised } \\ \text { CPB } & \text { Cricopharyngeal bar } \\ \text { IBM } & \text { Inclusion body myositis } \\ \text { IBMFRS } & \text { IBM Functional Rating Scale } \\ \text { IVIg } & \text { Intravenous immunoglobulin } \\ \text { FEES } & \text { Flexible endoscopic evaluation of swallowing } \\ \text { mOBFRS } & \text { Modified oculobulbarfacial respiratory score } \\ \text { PEG } & \text { Percutaneous endoscopic gastrostomy } \\ \text { PROs } & \text { Patient reported outcomes } \\ \text { RT-MRI } & \text { Real-time MRI } \\ \text { SIFA } & \text { Sporadic inclusion body myositis functioning assessment }\end{array}$


OBM Geriatrics 2021; 5(1), doi:10.21926/obm.geriatr.2101154

SWAL-QOL Swallowing quality of life questionnaire

VFS Videofluoroscopy

\section{Additional Materials}

The following additional materials are uploaded at the page of this paper.

1. Table S1: The modified Oculobulbar Facial Respiratory Score.

\section{Author Contributions}

Both authors, N.A.G. and T.M., conceived the idea as well as wrote and revised the manuscript into its final form.

\section{Competing Interests}

The authors have declared that no competing interests exist.

\section{References}

1. Weihl CC. Sporadic inclusion body myositis and other rimmed vacuolar myopathies. Continuum (Minneap Minn). 2019; 25: 1586-1598.

2. Rose MR. 188th ENMC international workshop: Inclusion body myositis, 2-4 December 2011, Naarden, The Netherlands. Neuromuscular Disord. 2013; 23: 1044-1055.

3. Glaubitz S, Zeng R, Schmidt J. New insights into the treatment of myositis. Ther Adv Musculoskeletal Dis. 2020; 12: 1759720X19886494.

4. Benveniste O, Guiguet M, Freebody J, Dubourg O, Squier W, Maisonobe T, et al. Long-term observational study of sporadic inclusion body myositis. Brain. 2011; 134: 3176-3184.

5. Lotz BP, Engel AG, Nishino H, Stevens JC, Litchy WJ. Inclusion body myositis: Observations in 40 patients. Brain. 1989; 112: 727-747.

6. Greenberg SA. Inclusion body myositis: Clinical features and pathogenesis. Nat Rev Rheumatol. 2019; 15: 257-272.

7. Oh TH, Brumfield KA, Hoskin TL, Stolp KA, Murray JA, Basford JR. Dysphagia in inflammatory myopathy: Clinical characteristics, treatment strategies, and outcome in 62 patients. Mayo Clin Proc. 2007; 82: 441-447.

8. Meyer A, Meyer N, Schaeffer M, Gottenberg JE, Geny B, Sibilia J. Incidence and prevalence of inflammatory myopathies: A systematic review. Rheumatology. 2015; 54: 50-63.

9. Callan A, Capkun G, Vasanthaprasad V, Freitas R, Needham M. A systematic review and metaanalysis of prevalence studies of sporadic inclusion body myositis. J Neuromuscul Dis. 2017; 4: 127137.

10. Needham M, Corbett A, Day T, Christiansen F, Fabian V, Mastaglia FL. Prevalence of sporadic inclusion body myositis and factors contributing to delayed diagnosis. J Clin Neurosci. 2008; 15 : 1350-1353.

11. Jones K, Pitceathly RD, Rose MR, McGowan S, Hill M, Badrising UA, et al. Interventions for dysphagia 
in long-term, progressive muscle disease. Cochrane Database Syst Rev. 2016; 2: CD004303.

12. Darrow DH, Hoffman HT, Barnes GJ, Wiley CA. Management of dysphagia in inclusion body myositis. Arch Otolaryngol Head Neck Surg. 1992; 118: 313-317.

13. Wintzen AR, Bots GT, De Bakker HM, Hulshof JH, Padberg GW. Dysphagia in inclusion body myositis . J Neurol Neurosurg Psychiatry. 1988; 51: 1542-1545.

14. Cox FM, Verschuuren JJ, Verbist BM, Niks EH, Wintzen AR, Badrising UA. Detecting dysphagia in inclusion body myositis. J Neurol. 2009; 256: 2009-2013.

15. Oh TH, Brumfield KA, Hoskin TL, Kasperbauer JL, Basford JR. Dysphagia in inclusion body myositis: Clinical features, management, and clinical outcome. Am J Phys Med Rehabil. 2008; 87: 883-889.

16. Felice KJ, North WA. Inclusion body myositis in connecticut: Observations in 35 patients during an 8-year period. Medicine. 2001; 80: 320-327.

17. Mulcahy KP, Langdon PC, Mastaglia F. Dysphagia in inflammatory myopathy: Self-report, incidence, and prevalence. Dysphagia. 2012; 27: 64-69.

18. Olthoff A, Carstens PO, Zhang S, von Fintel E, Friede T, Lotz J, et al. Evaluation of dysphagia by novel real-time MRI. Neurology. 2016; 87: 2132-2138.

19. Cox FM, Titulaer MJ, Sont JK, Wintzen AR, Verschuuren JJ, Badrising UA. A 12-year follow-up in sporadic inclusion body myositis: An end stage with major disabilities. Brain. 2011; 134: 3167-3175.

20. Shibata S, Izumi R, Hara T, Ohshima R, Nakamura N, Suzuki N, et al. Five-year history of dysphagia as a sole initial symptom in inclusion body myositis. J Neurol Sci. 2017; 381: 325.

21. Murata KY, Kouda K, Tajima F, Kondo T. A dysphagia study in patients with sporadic inclusion body myositis (s-IBM). Neurol Sci. 2012; 33: 765-770.

22. Peng A, Koffman BM, Malley JD, Dalakas MC. Disease progression in sporadic inclusion body myositis: Observations in 78 patients. Neurology. 2000; 55: 296-298.

23. Schrey A, Airas L, Jokela M, Pulkkinen J. Botulinum toxin alleviates dysphagia of patients with inclusion body myositis. J Neurol Sci. 2017; 380: 142-147.

24. Taira K, Yamamoto T, Mori-Yoshimura M, Fujita S, Oya Y, Nishino I, et al. Obstruction-related dysphagia in inclusion body myositis: Cricopharyngeal bar on videofluoroscopy indicates risk of aspiration. J Neurol Sci. 2020; 413: 116764.

25. Mohannak N, Pattison G, Hird K, Needham M. Dysphagia in patients with sporadic inclusion body myositis: Management challenges. Int J Gen Med. 2019; 12: 465-474.

26. Patel DA, Sharda R, Hovis KL, Nichols EE, Sathe N, Penson DF, et al. Patient-reported outcome measures in dysphagia: A systematic review of instrument development and validation. Dis Esophagus. 2017; 30: 1-23.

27. Zeng R, Schmidt J. Impact and management of dysphagia in inflammatory myopathies. Curr Rheumatol Rep. 2020; 22: 1-11

28. Langdon PC, Mulcahy K, Shepherd KL, Low VH, Mastaglia FL. Pharyngeal dysphagia in inflammatory muscle diseases resulting from impaired suprahyoid musculature. Dysphagia. 2012; 27: 408-417.

29. Taira K, Yamamoto T, Mori-Yoshimura M, Sajima K, Takizawa H, Shinmi J, et al. Cricopharyngeal bar on videofluoroscopy: High specificity for inclusion body myositis. J Neurol. 2020; 26: 1-9.

30. Nacci A, Ursino F, La Vela R, Matteucci FA, Mallardi V, Fattori BR. Fiberoptic endoscopic evaluation of swallowing (FEES): Proposal for informed consent. Acta Otorhinolaryngol Ital. 2008; 28: 206-211. 
31. Langmore SE. History of fiberoptic endoscopic evaluation of swallowing for evaluation and management of pharyngeal dysphagia: Changes over the years. Dysphagia. 2017; 32: 27-38.

32. Dziewas R, auf dem Brinke M, Birkmann U, Bräuer G, Busch K, Cerra F, et al. Safety and clinical impact of FEES-results of the FEES-registry. Neurol Res Pract. 2019; 1: 16.

33. Langmore SE. Evaluation of oropharyngeal dysphagia: Which diagnostic tool is superior? Curr Opin Otolaryngol Head Neck Surg. 2003; 11: 485-489.

34. Logemann JA, Rademaker AW, Pauloski BR, Ohmae Y, Kahrilas PJ. Normal swallowing physiology as viewed by videofluoroscopy and videoendoscopy. Folia Phoniatr Logop. 1998; 50: 311-319.

35. Postma GN, Butler SG, Belafsky PC, Halum SL. Normal pharyngeal and upper esophageal sphincter manometry. Ear Nose Throat J. 2004; 83: 809.

36. Zhang S, Olthoff A, Frahm J. Real-time magnetic resonance imaging of normal swallowing. J Magn Reson Imaging. 2012; 35: 1372-1379.

37. Van Den Engel-Hoek L, Lagarde M, Van Alfen N. Ultrasound of oral and masticatory muscles: Why every neuromuscular swallow team should have an ultrasound machine. Clin Anat. 2017; 30: 183193.

38. Cherin $P$, Pelletier $S$, Teixeira A, Laforet $P$, Simon A, Herson $S$, et al. Intravenous immunoglobulin for dysphagia of inclusion body myositis. Neurology. 2002; 58: 326.

39. Dalakas MC, Sonies B, Dambrosia J, Sekul E, Cupler E, Sivakumar K. Treatment of inclusion-body myositis with IVIg: A double-blind, placebo-controlled study. Neurology. 1997; 48: 712-716.

40. Dobloug C, Walle-Hansen R, Gran JT, Molberg $\varnothing$. Long-term follow-up of sporadic inclusion body myositis treated with intravenous immunoglobulin: A retrospective study of 16 patients. Clin Exp Rheumatol. 2012; 30: 838-842.

41. Cherin P, Delain JC, de Jaeger C, Crave JC. Subcutaneous immunoglobulin use in inclusion body myositis: A review of 6 cases. Case Rep Neurol. 2015; 7: 227-232.

42. Pars K, Garde N, Skripuletz T, Pul R, Dengler R, Stangel M. Subcutaneous immunoglobulin treatment of inclusion-body myositis stabilizes dysphagia. Muscle Nerve. 2013; 48: 838-839.

43. Langmore SE, Miller RM. Behavioral treatment for adults with oropharyngeal dysphagia. Arch Phys Med Rehabil. 1994; 75: 1154-1160.

44. Kahrilas PJ, Logemann JA, Krugler C, Flanagan E. Volitional augmentation of upper esophageal sphincter opening during swallowing. Am J Physiol Gastrointest Liver Physiol. 1991; 260: G450G456.

45. Mohannak N, Pattison G, Radich B, Hird K, Godecke E, Mastaglia F, et al. Exploring the efficacy of the expiratory muscle strength trainer to improve swallowing in inclusion body myositis: A pilot study. Neuromuscular Disord. 2020; 30: 294-300.

46. Schneider I, Pototschnig C, Thumfart WF, Eckel HE. Treatment of dysfunction of the cricopharyngeal muscle with botulinum A toxin: Introduction of a new, noninvasive method. Ann Otol Rhinol Laryngol. 1994; 103: 31-35.

47. Liu LW, Tarnopolsky M, Armstrong D. Injection of botulinum toxin a to upper esophageal sphincter for oropharyngeal dysphagia in two patients with inclusion body myositis. Can J Gastroenterol Hepatol. 2004; 18: 397-399.

48. Di Pede C, Masiero S, Bonsangue V, Ragona RM, Del Felice A. Botulinum toxin and rehabilitation 
treatment in inclusion body myositis for severe oropharyngeal dysphagia. Neurol Sci. 2016; 37: 1743-1745.

49. Parres C. Pharyngoesophageal dilation and botulinum toxin therapy for dysphagia in patients with inclusion body myositis. Neurology. 2015; 84: P3. 157.

50. Sanei-Moghaddam A, Kumar S, Jani P, Brierley C. Cricopharyngeal myotomy for cricopharyngeus stricture in an inclusion body myositis patient with hiatus hernia: A learning experience. BMJ Case Rep. 2013; 2013: bcr2012008058.

51. Benjamin Larman H, Salajegheh M, Nazareno R, Lam T, Sauld J, Steen H, et al. Cytosolic 5'nucleotidase 1A autoimmunity in sporadic inclusion body myositis. Ann Neurol. 2013; 73: 408-418.

52. Pluk H, van Hoeve BJ, van Dooren SH, Stammen-Vogelzangs J, van der Heijden A, Schelhaas HJ, et al. Autoantibodies to cytosolic 5'-nucleotidase $1 \mathrm{~A}$ in inclusion body myositis. Ann Neurol. 2013; 73: 397-407.

53. Goyal NA, Cash TM, Alam U, Enam S, Tierney P, Araujo N, et al. Seropositivity for NT5c1A antibody in sporadic inclusion body myositis predicts more severe motor, bulbar and respiratory involvement. J Neurol Neurosurg Psychiatry. 2016; 87: 373-378.

54. Lilleker JB, Rietveld A, Pye SR, Mariampillai K, Benveniste O, Peeters MT, et al. Cytosolic 5'nucleotidase $1 \mathrm{~A}$ autoantibody profile and clinical characteristics in inclusion body myositis. Ann Rheum Dis. 2017; 76: 862-868.

55. Araujo N, Wencel M, Medina E, et al. Feasibility and validation of modified oculobulbar facial respiratory score (mOBFRS) in amyotrophic lateral sclerosis. Neuromuscular Disord. 2019; 29: S67S68.

56. Araujo N, Wencel M, Medina E, Zhang L, Nguyen D, Habib A, et al. P. 05Feasibility and validation of modified oculobulbar facial respiratory score (mOBFRS) in sporadic inclusion body myositis. Neuromuscular Disord. 2019; 29: S42-S43.

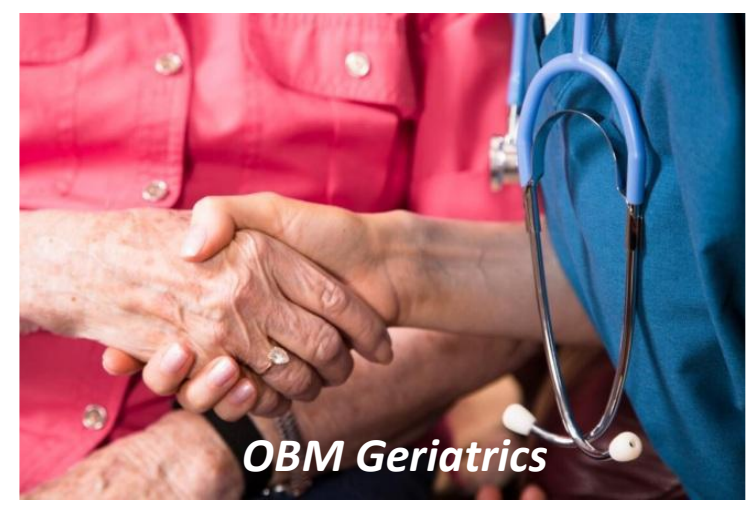

Enjoy OBM Geriatrics by:

1. Submitting a manuscript

2. Joining in volunteer reviewer bank

3. Joining Editorial Board

4. Guest editing a special issue

For more details, please visit: http://www.lidsen.com/journals/geriatrics 